\title{
Robust Low-rank Change Detection for Multivariate SAR Image Time Series
}

\author{
Ammar Mian, Antoine Collas, Arnaud Breloy, Member, IEEE, Guillaume Ginolhac, Senior Member, IEEE, \\ Jean-Philippe Ovarlez, Member, IEEE.
}

\begin{abstract}
This paper derives a new change detector for multivariate Synthetic Aperture Radar image time series. Classical statistical change detection methodologies based on covariance matrix analysis are usually built upon the Gaussian assumption, as well as an unstructured signal model. Both of these hypotheses may be inaccurate for high-dimension/resolution images, where the noise can be heterogeneous (non-Gaussian) and where the relevant signals usually lie in a low dimensional subspace (lowrank structure). These two issues are tackled by proposing a new Generalized Likelihood Ratio Test based on a robust (compound Gaussian) low-rank (structured covariance matrix) model. The interest of the proposed detector is assessed on two Synthetic Aperture Radar Image Time Series data set from UAVSAR.
\end{abstract}

Index Terms-Change detection, Synthetic Aperture Radar, time series, covariance matrix, low-rank, compound Gaussian.

\section{INTRODUCTION}

The analysis of Synthetic Aperture Radar (SAR) Image Time Series (ITS) has become a popular topic of research since it has many practical applications for Earth monitoring, such as disaster assessment, infrastructure monitoring or land-cover analysis. Over the past years, SAR-ITS have been made more widely available thanks to various missions such as Sentinel-1, TerraSAR-X, or UAVSAR. As a consequence, an active topic of research addresses the development of reliable automatic Change Detection (CD) methodologies in order to efficiently process this large amount of data. The CD problem is indeed challenging due to the lack of available ground truths, which does not allow applying supervised methods from the image processing literature. Moreover, it is well known that SAR images are subjected to speckle noise, which makes traditional optical approaches prone to high false alarm rates. In this case, unsupervised methodologies, often based on statistical tools, have yield interesting approaches in recent decades [2].

The CD problem can be seen as designing a metric that can assess for observed changes between pixels representing the

Ammar Mian is with Aalto university, Finland (email:ammar.mian@aalto.fi). Antoine Collas and Jean-Philippe Ovarlez are with SONDRA, CentraleSupelec, University of Paris Saclay, France (e-mails : antoine.collas, jean-philippe.ovarlez@centralesupelec.fr). JeanPhilippe Ovarlez is also with DEMR, ONERA, University of Paris Saclay, France (e-mail: jean-philippe.ovarlez@onera.fr). Arnaud Breloy is with LEME (EA4416), University Paris Nanterre, France (e-mail: abreloy@parisnanterre.fr). Guillaume Ginolhac is with LISTIC (EA3703), University Savoie Mont Blanc, France (e-mail: guillaume.ginolhac@univsmb.fr). This work was supported by ANR PHOENIX (ANR-15-CE23-0012) and ANR-ASTRID MARGARITA (ANR-17-ASTR-0015). A part of this work was presented at IGARSS 2019 [1]. The code and data sets used in this paper are available at https://github.com/AmmarMian/Robust-Low-Rank-CD. same location at different times. Among popular methodologies, Coherent Change Detection (CCD) [3] and the log-ratio operator [4] have received noticeable attention. However, these methodologies are limited to pairs of one-dimensional images, while current data-sets are generally multidimensional (using e.g., polarimetric or spectro-angular channels [5]), and gather multiple observation-times of the same scene. Multivariatedata oriented methodologies, i.e. that exploit the whole diversity, can improve the CD performance in this case. Notably, the local covariance matrix has been shown to be a relevant feature in order to assess for changes in multivariate SAR data [6]-[13].

Testing the similarity of covariances matrices between groups of observations is a well-established topic in the statistical literature [14]-[17], which has also been considered for CD in time-series in, e.g. [18], [19]. More specifically for SAR-ITS applications, various test statistics based on covariance matrix equality testing from Gaussian samples have been proposed within statistical detection framework [6]-[9], [11], or using information theory [12], [13] . A good review of these Gaussian detectors can be found in [10]. While these approaches offer good performance, they can nonetheless suffer from two issues encountered in high-dimension/resolution images: $i$ ) First, the Gaussian model has been shown to be inaccurate in recent radar clutter analysis due to the inherent heterogeneity of these images. In this case, Compound Gaussian models have been shown to provide a better fit to empirical measurements [20], [21], which is interesting to leverage in SAR images processing (e.g., in [22] for classification). In order to account for signals non-Gaussianity in SARITS, [23]-[25] proposed various Generalized Likelihood Ratio Tests (GLRTs) assuming a Compound Gaussian distribution. Specifically, [25] extended the Gaussian GLRT for covariance equality testing to the case of Compound Gaussian models, which was shown to yield improvements in terms of CD performance. $i i$ ) Second, the standard detectors are derived assuming unstructured covariance matrices, while the signal of interest usually lies in a low-dimensional subspace. This especially true for multivariate observations arising from radar systems, where a probabilistic principal component analysis model [26] can reflect a relevant assumption (see for example [27] and reference therein). Another example would be the case where only one polarization channel contains relevant information for change detection purpose. In this scope, [28] proposed to extend the GLRT approach to test for the equality of the parameters of low-rank structured covariance matrices.

To account for both issues, this paper proposes a new CD 
method based on both robust and LR models: we derive a GLRT for Compound Gaussian distributed observations that have a low-rank structured covariance matrix. The formulation of this test involves non-trivial optimization problems for which we tailor a practical block-coordinate descent algorithm. The proposed detector is then applied for CD on two SAR-ITS UAVSAR data set to demonstrate the interest of the approach for CD in SAR-ITS. This work constitutes an extension of the IGARSS conference paper [1], which was limited to the case of two images and did not present fully the algorithm derivations. The additional contributions are the following:

1) The full derivations for the general case are now provided.

2) Several side parameters (rank and noise floor variance) were assumed to be fixed in [1]. This extended version discusses how to deal with their estimation and presents experiments to test the robustness of the approach. Notably, it is shown that the performance of the proposed approach does not dramatically deteriorates if a slight error of the rank estimation is made.

3) Concerning the experiments on real data, we tested the proposed algorithm on new data sets: $i$ ) A time series (4 images) of a scene, where [1] contained an experiment restricted to two images of this scene; $i i$ ) An additional scene with 2 images.

The rest of the paper is organized as follows: Section II presents the general notations and the theoretical background (GLRT and useful distributions). Section III presents existing GLRTs for CD based on covariance matrix equality testing. Section IV details the proposed GLRT for CD and derives algorithms to evaluate it. Section V illustrates the performance of the proposed method on real data.

Notations: italic type indicates a scalar quantity, lower case boldface indicates a vector quantity and upper case boldface a matrix. The transpose conjugate operator is $H \cdot \operatorname{Tr}\{\cdot\}$ and $|\cdot|$ are respectively the trace and the determinant operators. $\operatorname{etr}\{\cdot\}$ is the exponential of trace operator. $\left\{w_{n}\right\}_{n=1}^{N}$ denotes the set of elements $w_{n}$, with $n \in \llbracket 1, N \rrbracket$. Definition of needed eigenvalue decomposition will be through the equality symbol $\stackrel{\mathrm{EVD}}{=} . \mathcal{H}_{p}^{++}$denotes the set of $p \times p$ Hermitian positive definite matrices. $\mathcal{H}_{p, R}^{++}$denotes the set of $p \times p$ Hermitian positive semi-definite matrices of rank $R$. $\propto$ stands for "proportional to". $\mathbf{x} \sim \mathcal{C N}(\boldsymbol{\mu}, \boldsymbol{\Sigma})$ is a complex-valued random Gaussian vector of mean $\boldsymbol{\mu}$ and CM $\boldsymbol{\Sigma}$.

\section{BACKGROUND}

\section{A. Multivariate SAR-ITS}

We consider a multidimensional time-series of $T$ multivariate SAR images as described in Figure 1. Each pixel of a SAR image at a given date $t$ corresponds to a vector of dimension $p$, denoted $\mathbf{x} \in \mathbb{C}^{p}$. The $p$ channels can correspond to a polarimetric diversity $(p=3)$, or to another kind of diversity such as a spectro-angular one, obtained through wavelet transforms [5]. The change detection process is applied using a local window around the pixel of interest, including $K$ pixels. Locally, the whole data set is denoted

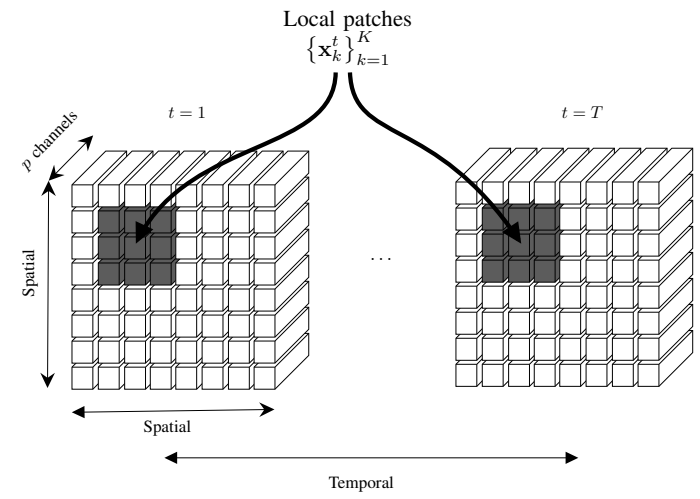

Fig. 1: Representation of $p$-variate SAR-ITS data set. The pixels highlighted in black correspond to the local observation window (here, $K=9$ ).

$\left\{\left\{\mathbf{x}_{k}^{t}\right\}_{k=1}^{K}\right\}_{t=1}^{T}$, which corresponds to the aggregation of all pixels at spatial indexes $k \in \llbracket 1, K \rrbracket$ and dates $t \in \llbracket 1, T \rrbracket$.

\section{B. Statistical CD with the GLRT}

For a given time $t$, the local observation $\left\{\mathbf{x}_{k}^{t}\right\}_{k=1}^{K}$ is assumed to be distributed according to a fixed parametric distribution, of parameter $\boldsymbol{\theta}_{t}$. The corresponding likelihood is denoted $\mathcal{L}\left(\left\{\mathbf{x}_{k}^{t}\right\}_{k=1}^{K} \mid \boldsymbol{\theta}_{t}\right)$. The parameters $\left\{\boldsymbol{\theta}_{t}\right\}$ are a feature that characterizes the local data at each date $t$ : if a local change occurs, this parameter is expected to vary. The CD problem can thus be formulated as a binary hypothesis test:

$$
\begin{cases}\mathrm{H}_{0}: & \boldsymbol{\theta}_{i}=\boldsymbol{\theta}_{0}, \forall i \in \llbracket 1, T \rrbracket \quad \text { (no change) }, \\ \mathrm{H}_{1}: & \boldsymbol{\theta}_{i} \neq \boldsymbol{\theta}_{j}, \text { for } i \neq j \quad \text { (change). }\end{cases}
$$

Notice that we consider an omnibus CD problem, i.e. we do not test for a change at a specific date. In this work, the sequential test focusing on the date $t_{0}$ :

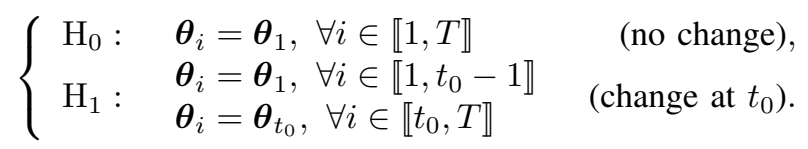

can be trivially recasted as an omnibus one with $T=2$ by re-partitioning the data set. However, this property is not always true depending on the chosen distribution and set of parameters.

In order to derive a metric of decision, we consider the use of GLRT. This test consists in computing the following quantity:

$$
\hat{\Lambda}_{\mathrm{GLRT}}=\frac{\max _{\left\{\boldsymbol{\theta}_{t}\right\}_{t=1}^{T}} \prod_{t=1}^{T} \mathcal{L}^{\mathrm{H}_{1}}\left(\left\{\mathbf{x}_{k}^{t}\right\}_{k=1}^{K} \mid \boldsymbol{\theta}_{t}\right)}{\max _{\boldsymbol{\theta}_{0}} \prod_{t=1}^{T} \mathcal{L}^{\mathrm{H}_{0}}\left(\left\{\mathbf{x}_{k}^{t}\right\}_{k=1}^{K} \mid \boldsymbol{\theta}_{0}\right)},
$$

where $\mathcal{L}^{\mathrm{H}_{1}}$ (resp. $\mathcal{L}^{\mathrm{H}_{0}}$ ) denotes the likelihood function and $\left\{\boldsymbol{\theta}_{t}\right\}_{t=1}^{T}$ (resp. $\boldsymbol{\theta}_{0}$ ) corresponds to the parameters of the distribution, both under $\mathrm{H}_{1}$ (resp. $\mathrm{H}_{0}$ ). Hence, to develop efficient detectors, the problem remains to select an assumed distribution (and corresponding parameters) that accurately reflects the behavior of the data. It is also worth mentioning 
that, depending on the chosen model, the evaluation of the GLRT may lead to complex optimization problems.

\section{Compound Gaussian distributions}

The Gaussian assumption is the most widely used in multivariate SAR image applications. As each pixel value can be the sum of the contribution of many scatterers, this assumption is indeed well motivated by the central limit theorem. For SAR images, the mean is also classically assumed to be zero due to the multiplicative nature of speckle noise and will be thus omitted in this work. A complex $p$-vector $\mathbf{x} \in \mathbb{C}^{p}$ follows a zero-mean multivariate circular complex Gaussian distribution, denoted $\mathrm{x} \sim \mathcal{C N}(\mathbf{0}, \boldsymbol{\Sigma})$, if it has the Probability Density Function (PDF)

$$
f_{\mathbf{x}}^{\mathcal{C N}}(\mathbf{x} \mid \boldsymbol{\Sigma})=\pi^{-p}|\boldsymbol{\Sigma}|^{-1} \exp \left(-\mathbf{x}^{H} \boldsymbol{\Sigma}^{-1} \mathbf{x}\right) .
$$

where $\boldsymbol{\Sigma} \in \mathcal{H}_{p}^{++}$is the covariance matrix, i.e. $\mathbb{E}\left[\mathbf{x x}^{H}\right]=\boldsymbol{\Sigma}$. A set of observations $\left\{\mathbf{x}_{k}\right\}_{k=1}^{K}$ has then the likelihood

$$
\mathcal{L}_{\mathcal{C N}}\left(\left\{\mathbf{x}_{k}\right\}_{k=1}^{K} \mid \boldsymbol{\Sigma}\right) \propto|\boldsymbol{\Sigma}|^{-K} \operatorname{etr}\left\{-\boldsymbol{\Sigma}^{-1} \mathbf{X X}^{H}\right\}
$$

with $\mathbf{X}=\left[\mathbf{x}_{1}, \ldots, \mathbf{x}_{K}\right]$.

Nevertheless, the Gaussian assumption can be inaccurate when it comes to model high-resolution images or heterogeneous areas. In this case, distributions that can account for heavy-tails, such as the compound Gaussian ones, offer a better fit [21]. A vector $\mathbf{x} \in \mathbb{C}^{p}$ follows a zero-mean multivariate complex compound Gaussian distribution, denoted $\mathbf{x} \sim \mathcal{C G}\left(\mathbf{0}, \boldsymbol{\Sigma}, f_{\tau}\right)$, if it admits the stochastic representation

$$
\mathbf{x} \stackrel{d}{=} \sqrt{\tau} \mathbf{n},
$$

with $\mathbf{n} \sim \mathcal{C N}(\mathbf{0}, \boldsymbol{\Sigma})$, and where $\tau$ is a positive random scalar, independent from $\mathbf{n}$, with PDF $f_{\tau}($.$) . Compound Gaussian$ distributions encompass a large family of standard multivariate ones, depending on the assumed $\mathrm{PDF} f_{\tau}($.$) [21]. In order to$ design a process that is robust to all these distributions, we can assume that $\left\{\tau_{k}\right\}$ are unknown deterministic variables. For a data set $\left\{\mathbf{x}_{k}\right\}$, this assumption leads to $\mathbf{x}_{k} \mid \tau_{k} \sim \mathcal{C N}\left(\mathbf{0}, \tau_{k} \boldsymbol{\Sigma}\right)$, with the likelihood function

$$
\begin{aligned}
\mathcal{L}_{\mathcal{C G}}\left(\left\{\mathbf{x}_{k}\right\}_{k=1}^{K} \mid \boldsymbol{\Sigma},\left\{\tau_{k}\right\}_{k=1}^{K}\right) \\
\propto \prod_{k=1}^{K}\left|\tau_{k} \boldsymbol{\Sigma}\right|^{-1} \exp \left(-\mathbf{x}_{k}^{H}\left(\tau_{k} \boldsymbol{\Sigma}\right)^{-1} \mathbf{x}_{k}\right)
\end{aligned}
$$

\section{CD WITH GLRTS BASED ON COVARIANCE MATRIX}

For SAR-ITS, the CD problem can be reformulated as a hypothesis test on equality of the covariance matrices. The rationale behind this is that a local change is translated by a change in the second-order statistics of the observations. To perform this test, the GLRT approach has been successfully applied in numerous works [6]-[13], [25], [28]. The following subsections recall existing GLRTs (depending on the assumed model) that have been applied to CD in SAR-ITS.

\section{A. Gaussian $C D$}

Assuming Gaussian distributed samples, the $\mathrm{CD}$ can be performed by testing a change in the covariance matrix [6],
[7]. The corresponding GLRT, denoted $\hat{\Lambda}_{\mathrm{G}}$, corresponds to (1) and (3) with the following distribution/parameters:

$$
\text { Model: } \quad \mathbf{x}_{k}^{t} \sim \mathcal{C N}\left(\mathbf{0}, \boldsymbol{\Sigma}_{t}\right)
$$$$
\text { Likelihood in (5) }
$$

Param.: $\mathrm{H}_{0}: \boldsymbol{\theta}_{0}=\boldsymbol{\Sigma}_{0}$

$$
\mathrm{H}_{1}:\left\{\boldsymbol{\theta}_{t}\right\}_{t=1}^{T}=\left\{\boldsymbol{\Sigma}_{t}\right\}_{t=1}^{T}
$$

this test has well established statistical properties (cf. eg. [10]) and admits the closed-form expression

$$
\hat{\Lambda}_{\mathrm{G}}=\frac{\left|\hat{\boldsymbol{\Sigma}}_{0}^{\mathrm{SCM}}\right|}{\prod_{t=1}^{T}\left|\hat{\boldsymbol{\Sigma}}_{t}^{\mathrm{SCM}}\right|^{1 / T}},
$$

involving the following Sample Covariance Matrices (SCM) $\hat{\boldsymbol{\Sigma}}_{0}^{\mathrm{SCM}}=\frac{1}{T K} \sum_{t, k} \mathbf{x}_{k}^{t}\left(\mathbf{x}_{k}^{t}\right)^{H}, \hat{\boldsymbol{\Sigma}}_{t}^{\mathrm{SCM}}=\frac{1}{K} \sum_{k} \mathbf{x}_{k}^{t}\left(\mathbf{x}_{k}^{t}\right)^{H}$.

\section{B. Compound Gaussian CD}

As stated previously, the Gaussian assumption is no longer valid for high-resolution, or heterogeneous SAR images [20]. This mismodeling induces a strong reduction of the CD performance when using $\hat{\Lambda}_{\mathrm{G}}$, notably caused by the inaccuracy of the SCM computed from non-Gaussian observations. This issue can be alleviated by assuming a compound Gaussian model, as described in section II-C. Under this assumption, the CD can be performed by testing a change in both the covariance matrix and the texture parameters [25]. The corresponding GLRT, denoted $\hat{\Lambda}_{\mathrm{CG}}$, corresponds to (1) and (3) with the following distribution/parameters:

$$
\begin{array}{cl}
\text { Model: } & \mathbf{x}_{k}^{t} \sim \mathcal{C N}\left(\mathbf{0}, \tau_{k}^{t} \boldsymbol{\Sigma}_{t}\right) \\
& \text { Likelihood in }(7) \\
\text { Param.: } & \mathrm{H}_{0}: \boldsymbol{\theta}_{0}=\left\{\boldsymbol{\Sigma}_{0},\left\{\tau_{k}^{0}\right\}_{k=1}^{K}\right\} \\
& \mathrm{H}_{1}:\left\{\boldsymbol{\theta}_{t}\right\}_{t=1}^{T}=\left\{\boldsymbol{\Sigma}_{t},\left\{\tau_{k}^{t}\right\}_{k=1}^{K}\right\}_{t=1}^{T}
\end{array}
$$

The evaluation of the quantity $\hat{\Lambda}_{\mathrm{CG}}$ involves fixed-point equations that can be computed numerically. A study and generalizations (testing for textures or covariance matrices individually) of this approach can be found in [25].

\section{Low-rank Gaussian CD}

The performance of the aforementioned CD methods is tightly linked to the accuracy of the covariance matrix estimation. The general rule-of-thumb requires $K>2 p$ samples in order to obtain a correct estimation [29]. In order to lower $K$ (i.e., reduce the local window size), some prior knowledge on the covariance structure can be leveraged to reduce the dimension of the estimation problem [30]-[33]. Due to signals lying in a lower dimensional subspace, a very common structure in radar applications is the low-rank one, i.e.

$$
\boldsymbol{\Sigma}=\boldsymbol{\Sigma}_{R}+\sigma^{2} \mathbf{I}
$$

where $\boldsymbol{\Sigma}_{R}$ belongs to the set of Hermitian positive semidefinite matrices of rank $R$, denoted $\mathcal{H}_{p, R}^{+}$. A Gaussian GLRT that accounts for this prior knowledge (with known $\sigma^{2}$ ), 
denoted $\hat{\Lambda}_{\mathrm{LRG}}$, can be formulated according to (1) and (3) with the following distribution/parameters:

$$
\begin{array}{cl}
\text { Model: } & \mathbf{x}_{k}^{t} \sim \mathcal{C N}\left(\mathbf{0}, \boldsymbol{\Sigma}_{R}^{t}+\sigma^{2} \mathbf{I}\right) \\
& \text { Likelihood in (5) } \\
\text { Param.: } & \mathrm{H}_{0}: \boldsymbol{\theta}_{0}=\left\{\boldsymbol{\Sigma}_{R}^{0}\right\} \\
& \mathrm{H}_{1}:\left\{\boldsymbol{\theta}_{t}\right\}_{t=1}^{T}=\left\{\boldsymbol{\Sigma}_{R}^{t}\right\}_{t=1}^{T}
\end{array}
$$

Several generalizations (testing for specific parameters individually) of this approach, and algorithms to compute the corresponding quantities, can be found in [28], [34]. When compared to $\hat{\Lambda}_{\mathrm{G}}, \hat{\Lambda}_{\mathrm{LRG}}$ offers a gain in terms of performance and spatial resolution. However, the robustness properties of $\hat{\Lambda}_{\mathrm{CG}}$ are lost, that motivates the present work.

\section{PRoposed Detector}

\section{A. Low-rank compound Gaussian CD}

In this paper, we propose to combine the advantages of both the low-rank structure and the CG distribution. Thus, we consider a model of CG distributed samples (cf. Section II-C) with a LR structured covariance matrix as in (11). The corresponding GLRT for CD, denoted $\hat{\Lambda}_{\mathrm{LRCG}}$, corresponds to (1) and (3) with the following distribution/parameters:

Model: $\quad \mathbf{x}_{k}^{t} \sim \mathcal{C N}\left(\mathbf{0}, \tau_{k}^{t}\left(\boldsymbol{\Sigma}_{R}^{t}+\sigma_{t}^{2} \mathbf{I}\right)\right)$ Likelihood in (7)

Param.: $\quad \mathrm{H}_{0}: \boldsymbol{\theta}_{0}=\left\{\boldsymbol{\Sigma}_{R}^{0}, \sigma_{0}^{2},\left\{\tau_{k}^{0}\right\}_{k=1}^{K}\right\}$

$$
\mathrm{H}_{1}:\left\{\boldsymbol{\theta}_{t}\right\}_{t=1}^{T}=\left\{\boldsymbol{\Sigma}_{R}^{t}, \sigma_{t}^{2},\left\{\tau_{k}^{t}\right\}_{k=1}^{K}\right\}_{t=1}^{T}
$$

Here, the test accounts for a possible change of both the covariance matrix and the textures between acquisitions, as it was shown to be the most relevant approach for SAR-ITS [25]. Also note that we consider a more general model than (12), where the variance $\sigma_{t}^{2}$ is unknown and can change at each date $t$.

\section{B. Computation of $\Lambda_{\mathrm{LRCG}}$}

Evaluating $\Lambda_{\mathrm{LRCG}}$ according to the generic equation (3) requires to compute:

$$
\hat{\Lambda}_{\mathrm{LRCG}}=\frac{\mathcal{L}_{L R C G}^{\mathrm{H}_{1}}\left(\left\{\left\{\mathbf{x}_{k}^{t}\right\}_{k=1}^{K}\right\}_{t=1}^{T} \mid \hat{\boldsymbol{\theta}}_{\mathrm{LRCG}}^{\mathrm{H}_{1}}\right)}{\mathcal{L}_{L R C G}^{\mathrm{H}_{0}}\left(\left\{\left\{\mathbf{x}_{k}^{t}\right\}_{k=1}^{K}\right\}_{t=1}^{T} \mid \hat{\boldsymbol{\theta}}_{\mathrm{LRCG}}^{\mathrm{H}_{0}}\right)},
$$

where $\mathcal{L}_{L R C G}^{\mathrm{H}_{0}}$ and $\mathcal{L}_{L R C G}^{\mathrm{H}_{1}}$ are the likelihood (derived from (7)) under $\mathrm{H}_{0}$ and $\mathrm{H}_{1}$, and where

$$
\begin{aligned}
& \hat{\boldsymbol{\theta}}_{\mathrm{LRCG}}^{\mathrm{H}_{0}}=\left\{\hat{\boldsymbol{\Sigma}}_{R}^{0}, \hat{\sigma}_{0}^{2},\left\{\hat{\tau}_{k}^{0}\right\}_{k=1}^{K}\right\}, \\
& \hat{\boldsymbol{\theta}}_{\mathrm{LRCG}}^{\mathrm{H}_{1}}=\left\{\hat{\boldsymbol{\Sigma}}_{R}^{t}, \hat{\sigma}_{t}^{2},\left\{\hat{\tau}_{k}^{t}\right\}_{k=1}^{K}\right\}_{t=1}^{T}
\end{aligned}
$$

are the maximum likelihood estimators under $\mathrm{H}_{0}$ and $\mathrm{H}_{1}$, respectively.

1) Maximum likelihood under $\mathrm{H}_{1}$ : Under $\mathrm{H}_{1}$, the likelihood is separable in $t$. For a fixed $t$, the maximum likelihood

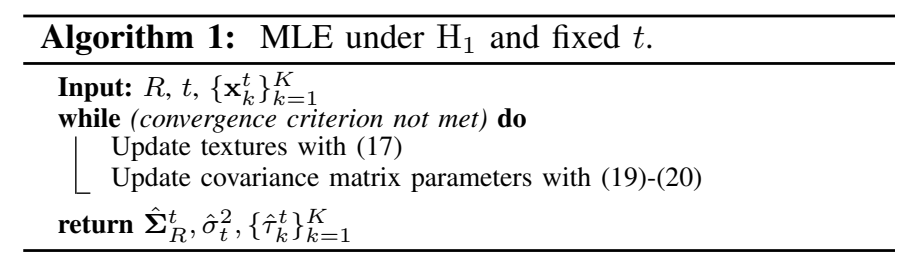

estimation of the unknown parameters $\left\{\hat{\boldsymbol{\Sigma}}_{R}^{t}, \hat{\sigma}_{t}^{2},\left\{\hat{\tau}_{k}^{t}\right\}_{k=1}^{K}\right\}$ consists in solving the problem

$$
\begin{array}{cl}
\underset{\left\{\tau_{k}^{t}, \boldsymbol{\Sigma}_{k}^{t}\right\}_{k=1}^{K}, \boldsymbol{\Sigma}_{R}^{t}, \sigma_{t}^{2}}{\operatorname{maximize}} & \sum_{k=1}^{K} \log \left(\mathcal{L}_{\mathrm{H}_{1}}^{t}\left(\mathbf{x}_{k}^{t} \mid \boldsymbol{\Sigma}_{k}^{t}\right)\right) \\
\text { subject to } & \boldsymbol{\Sigma}_{k}^{t}=\tau_{k}^{t}\left(\boldsymbol{\Sigma}_{R}^{t}+\sigma_{t}^{2} \mathbf{I}\right) \\
& \boldsymbol{\Sigma}_{R}^{t} \succcurlyeq \mathbf{0}, \text { and } \operatorname{rank}\left(\boldsymbol{\Sigma}_{R}^{t}\right)=R \\
& \sigma_{t}^{2}>0, \text { and } \tau_{k}^{t}>0, \forall k
\end{array}
$$

where $\mathcal{L}_{\mathrm{H}_{1}}^{t}\left(\mathbf{x}_{k}^{t} \mid \boldsymbol{\Sigma}_{k}\right)$ reads directly from (4). The solution to this problem can not be obtained in closed form, but the following sections derive practical block-coordinate descent algorithm in order to evaluate it. The algorithm is summed up in the box Algorithm 1.

- Update of the textures $\left(\mathrm{H}_{1}\right)$ : Assuming a fixed covariance matrix $\boldsymbol{\Sigma}_{R}^{t}+\sigma_{t}^{2} \mathbf{I}$, the maximum likelihood estimator of the texture parameters is obtained in closed form [35], with

$$
\hat{\tau}_{k}^{t}=\frac{1}{p}\left(\mathbf{x}_{k}^{t}\right)^{H}\left(\boldsymbol{\Sigma}_{R}^{t}+\sigma_{t}^{2} \mathbf{I}\right)^{-1} \mathbf{x}_{k}^{t}
$$

- Update of the covariance matrix parameters $\left(\mathrm{H}_{1}\right)$ : Assuming fixed textures $\left\{\tau_{k}^{t}\right\}_{k=1}^{K}$, the problem (16) can be re-expressed as:

$$
\begin{array}{cl}
\underset{\boldsymbol{\Sigma}_{t}, \boldsymbol{\Sigma}_{R}^{t}, \sigma_{t}^{2}}{\operatorname{minimize}} & \log \left|\boldsymbol{\Sigma}_{t}\right|+\operatorname{Tr}\left\{\tilde{\mathbf{S}}_{t} \boldsymbol{\Sigma}_{t}^{-1}\right\} \\
\text { subject to } & \boldsymbol{\Sigma}_{t}=\boldsymbol{\Sigma}_{R}^{t}+\sigma_{t}^{2} \mathbf{I} \\
& \boldsymbol{\Sigma}_{R}^{t} \succcurlyeq \mathbf{0}, \text { and } \operatorname{rank}\left(\boldsymbol{\Sigma}_{R}^{t}\right)=R \\
& \sigma_{t}^{2}>0
\end{array}
$$

with $\tilde{\mathbf{S}}_{t}=(1 / K) \sum_{k=1}^{K} \mathbf{x}_{k}^{t}\left(\mathbf{x}_{k}^{t}\right)^{H} / \tau_{k}^{t}$. The solution is given in [26], and leads to the update

$$
\boldsymbol{\Sigma}_{t}=\mathbf{U} \operatorname{diag}(\tilde{\mathbf{d}}) \mathbf{U}^{H} \triangleq \mathcal{T}_{\mathbf{R}}\left(\tilde{\mathbf{S}}_{t}\right)
$$

defined through the operator $\mathcal{T}_{\mathrm{R}}$, with

$$
\begin{aligned}
& \tilde{\mathbf{S}}_{t} \stackrel{\mathrm{EVD}}{=} \mathbf{U} \operatorname{diag}(\mathbf{d}) \mathbf{U}^{H} \\
& \mathbf{d}=\left[d_{1}, \ldots, d_{R}, d_{R+1}, \ldots, d_{p}\right] \\
& \tilde{\mathbf{d}}=\left[d_{1}, \ldots, d_{R}, \hat{\sigma}_{t}^{2}, \ldots, \hat{\sigma}_{t}^{2}\right] \\
& \hat{\sigma}_{t}^{2}=\frac{1}{p-R} \sum_{r=R+1}^{p} d_{r} .
\end{aligned}
$$

Remark: If the noise variance is assumed to be known and equal to $\sigma^{2}$, the solution of the modified problem (with constraint $\left.\operatorname{rank}\left(\boldsymbol{\Sigma}_{R}^{t}\right) \leq R\right)$ is given in [27], and consists in replacing $\tilde{\mathbf{d}}$ by

$$
\overline{\mathbf{d}}=\left[\max \left(d_{1}, \sigma^{2}\right), \ldots, \max \left(d_{R}, \sigma^{2}\right), \sigma^{2}, \ldots, \sigma^{2}\right] .
$$




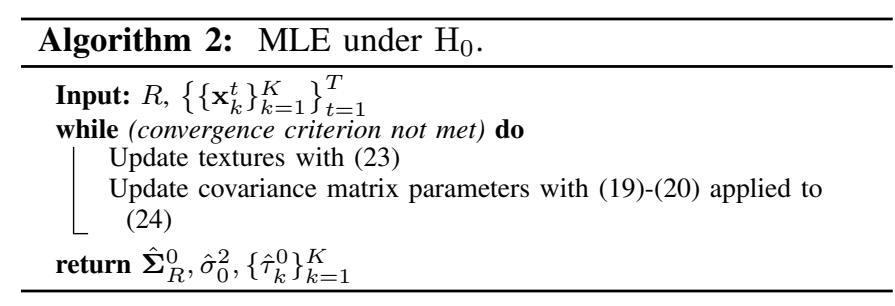

2) Maximum likelihood under $\mathrm{H}_{0}$ :

$$
\begin{array}{cl}
\underset{\left\{\tau_{k}^{0}, \boldsymbol{\Sigma}_{k}^{0}\right\}_{k=1}^{K}, \boldsymbol{\Sigma}_{R}^{0}, \sigma_{0}^{2}}{\operatorname{maximize}} & \sum_{t=1}^{T} \sum_{k=1}^{K} \log \left(\mathcal{L}_{\mathrm{H}_{0}}^{t}\left(\mathbf{x}_{k}^{t} \mid \boldsymbol{\Sigma}_{k}^{0}\right)\right) \\
\text { subject to } & \boldsymbol{\Sigma}_{k}^{0}=\tau_{k}^{0}\left(\boldsymbol{\Sigma}_{R}^{0}+\sigma_{0}^{2} \mathbf{I}\right) \\
& \boldsymbol{\Sigma}_{R}^{0} \succcurlyeq \mathbf{0}, \text { and } \operatorname{rank}\left(\boldsymbol{\Sigma}_{R}^{0}\right)=R \\
& \sigma_{0}^{2}>0, \text { and } \tau_{k}^{0}>0, \forall k
\end{array}
$$

This problem can be solved as the one in (16), with some modifications due to the likelihood function. The differences are detailed below and summed up in the box algorithm 2 .

- Update of the textures $\left(\mathrm{H}_{0}\right)$ : Assuming a fixed covariance matrix $\boldsymbol{\Sigma}^{0}=\boldsymbol{\Sigma}_{R}^{0}+\sigma_{0}^{2} \mathbf{I}$, the maximum likelihood estimator of the texture parameters is obtained in closed form [25], with

$$
\hat{\tau}_{k}^{0}=\frac{1}{T p} \sum_{t=1}^{T}\left(\mathbf{x}_{k}^{t}\right)^{H}\left(\boldsymbol{\Sigma}_{R}^{0}+\sigma_{0}^{2} \mathbf{I}\right)^{-1} \mathbf{x}_{k}^{t}
$$

- Update of the covariance matrix parameters $\left(\mathrm{H}_{0}\right)$ : Assuming fixed textures, the update problem can be reformulated as in (18), using

$$
\tilde{\mathbf{S}}_{0}=\frac{1}{K T} \sum_{t=1}^{T} \sum_{k=1}^{K} \frac{\mathbf{x}_{k}^{t}\left(\mathbf{x}_{k}^{t}\right)^{H}}{\tau_{k}^{0}}
$$

instead of $\tilde{\mathbf{S}}_{t}$. The solution is then obtained as in (19) and (20), and yields $\boldsymbol{\Sigma}_{0} \triangleq \mathcal{T}_{\mathrm{R}}\left(\tilde{\mathbf{S}}_{0}\right)$.

3) Note on the convergence: Both algorithms 1 and 2 appear as technical variations around the one of $[32$, Sec. V.A.], which derives a Majorization-Minimization algorithm for an objective that corresponds to the concentrated likelihood (substituting the maximum likelihood estimates of $\left\{\tau_{k}\right\}_{k=1}^{K}$ ) under $\mathrm{H}_{1}$. Some convergence properties can thus be deduced from [32]. The proposed algorithms are block-coordinates descent with a unique global solution at each step. Since the problem is not convex, this does not offer a theoretical guarantee on the convergence in terms of variables (though it is experienced in practice). However, it ensures a monotonic increment of the likelihood and, thus a convergence in terms of objective value. This property is essential since we are mostly interested in the evaluation of (14).

\section{Selecting the rank $R$}

The proposed detector requires to assume a rank $R$. In practice, this rank can be pre-estimated (either globally or locally) by using rank estimation methods from the literature, such as information theory-based ones [36]. In this paper, we consider a simple approach, i.e. fixing a global rank by analyzing the eigenvalues of the total sample covariance over the ITS. We will also test the robustness of the approach to the choice of $R$.

\section{NUMERICAL SIMULATIONS}

The performance of the proposed change detector $\Lambda_{\mathrm{LRCG}}$ is tested on a SAR ITS dataset, and assessed with ROC curves (displaying the probability of detection versus the false alarm rate). As a mean to assess the effectiveness of combining LR structure with a robust model, it is compared to the following detectors: $i$ ) the classic Gaussian detector proposed in [6], [10], denoted $\left.\hat{\Lambda}_{G} ; i i\right)$ the LR Gaussian detector of [28], denoted $\hat{\Lambda}_{L R G} ;$ iii) the compound Gaussian detector proposed in [25], denoted $\hat{\Lambda}_{C G}$.

\section{A. Datasets description}

The SAR ITS data set is taken from UAVSAR (courtesy of NASA/JPL-Caltech). Two scenes with respectively 4 and 2 images are used ${ }^{1}$. They are displayed in Figure 2 for the scene 1 (4 images) and 3 for the scene 2 ( 2 images). The CD ground truths are collected from [12], [37] and are shown in figures 4. Table I gives an overall perspective of the scenes used in the study. The SAR images correspond to full-polarization data with a resolution of $1.67 \mathrm{~m}$ in range and $0.6 \mathrm{~m}$ in azimuth. Since the scatterers present in this scene exhibit an interesting spectro-angular behavior, each polarization of these images has been subjected to the wavelet transform presented in [5], allowing to obtain images of dimension $p=12$.

The rank $R$ is chosen according to Figure 5, which displays the eigenvalues of the total sample covariance matrix. For this data set, $R=3$ appears to be an interesting value to separate signal from noise components. Notably, this rank gathers $81 \%$ of the total variance.

\section{B. Results}

1) Comparison between four methods: Figure 6 displays the outputs of the 4 detectors applied to scene 1 . From visual inspection, the levels of the false alarms appear lower for the low-rank based detectors. Figure 7 confirms this insight, and also assesses that the proposed method achieves the best performance in terms of probability of detection versus false alarm rate. For the scene 2, the same conclusions can be drawn from Figure 9 (detectors output) and Figure 10 (ROC curves). Finally, Figures 8 and 11 display the thresholded detection maps of each scene so that the probability of false alarm is identical for all detectors (set to 10\%). These confirm the previous conclusions by showing that the proposed method improves the detection results at the edge of several zones (circled in red in the figure of $\hat{\Lambda}_{\mathrm{LRCG}}$ ).

2) Robustness to rank selection: Figure 12 displays the ROC curves of $\Lambda_{\text {LRCG }}$ on the scene 1 for three different values of the rank $R$. It is interesting to notice that these curves do not vary significantly with respect to this parameter. Therefore, we can expect that a slight error in the rank estimation will not lead to a significant drop in CD performance.

\footnotetext{
${ }^{1}$ The scene 1 reduced to $T=2$ is studied in [1]
} 
TABLE I: Description of SAR data used

\begin{tabular}{|c|c|c|c|c|c|c|c|}
\hline Dataset & $\mathrm{Url}$ & Resolution & Scene & $p$ & $T$ & Size & $\begin{array}{l}\text { Coordinates } \\
\text { (top-left px) }\end{array}$ \\
\hline \multirow[t]{2}{*}{$\begin{array}{l}\text { UAVSAR } \\
\text { SanAnd_26524_03 Segment } 4 \\
\text { April 23, 2009 - May 15, } 2011\end{array}$} & \multirow[t]{2}{*}{ https://uavsar.jpl.nasa.gov } & \multirow[t]{2}{*}{$\begin{array}{l}\mathrm{Rg}: 1.67 \mathrm{~m} \\
\mathrm{Az}: 0.6 \mathrm{~m}\end{array}$} & Scene 1 & 12 & 4 & $2360 \times 600 \mathrm{px}$ & $\begin{array}{l}{[\mathrm{Rg}, \mathrm{Az}]=} \\
{[2891,28891]}\end{array}$ \\
\hline & & & Scene 2 & 12 & 2 & $2300 \times 600 \mathrm{px}$ & $\begin{array}{l}{[\mathrm{Rg}, \mathrm{Az}]=} \\
{[3236,25601]}\end{array}$ \\
\hline
\end{tabular}

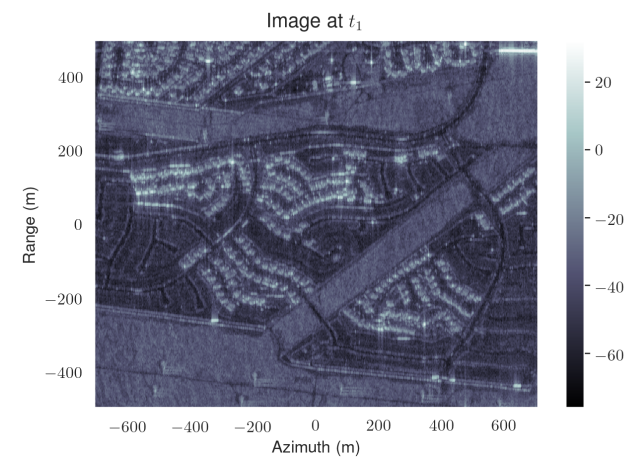

(a) $\mathrm{t}=1$

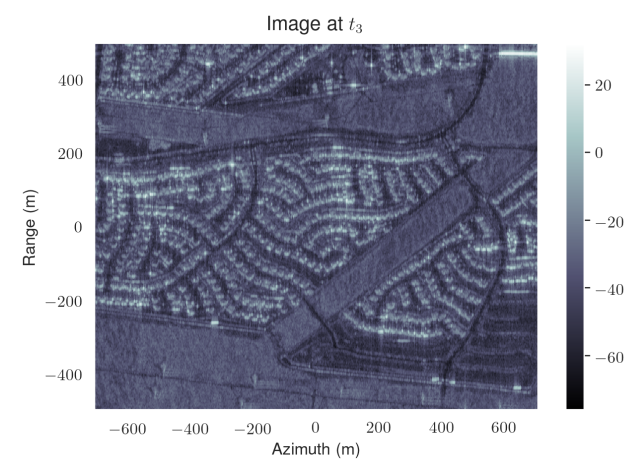

(c) $t=3$

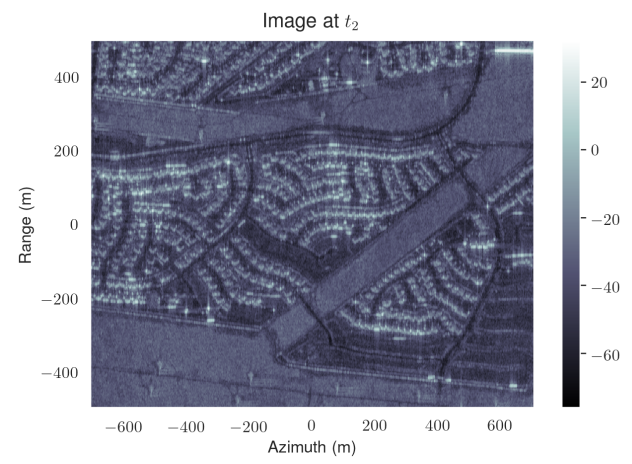

(b) $\mathrm{t}=2$

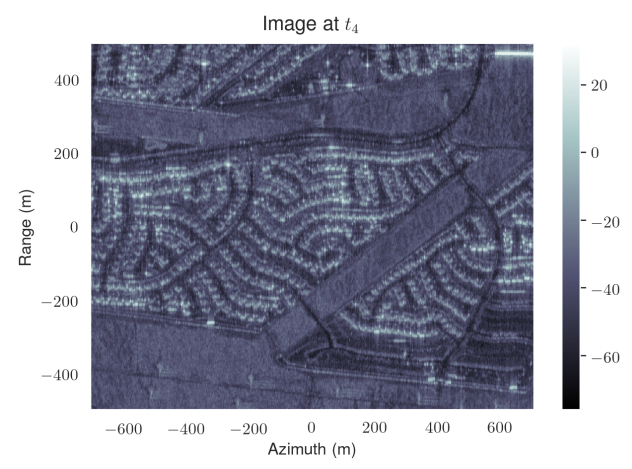

(d) $t=4$

Fig. 2: UAVSAR Dataset used in this study for the scene 1. Four dates are available between April 23, 2009 and May 15, 2011.

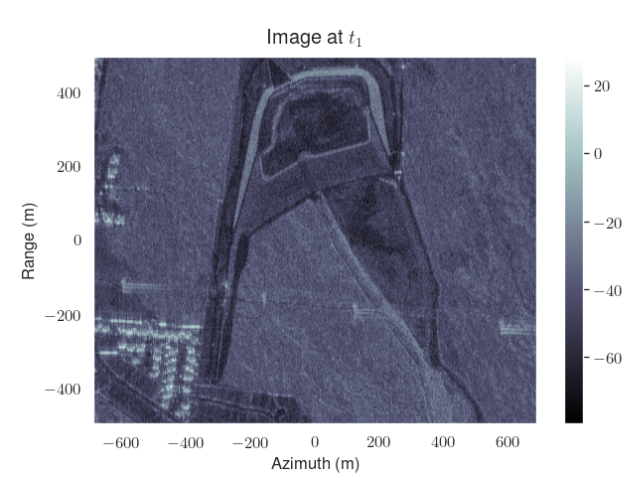

(a) $\mathrm{t}=1$

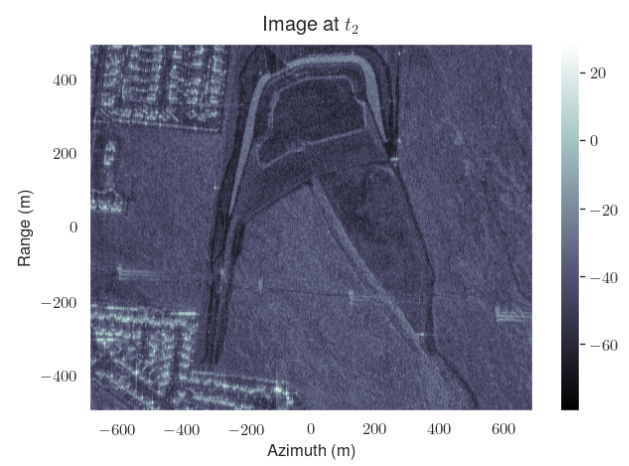

(b) $\mathrm{t}=2$

Fig. 3: UAVSAR Dataset used in this study for the scene 2. Four dates are available between April 23, 2009 and May 15, 2011. 


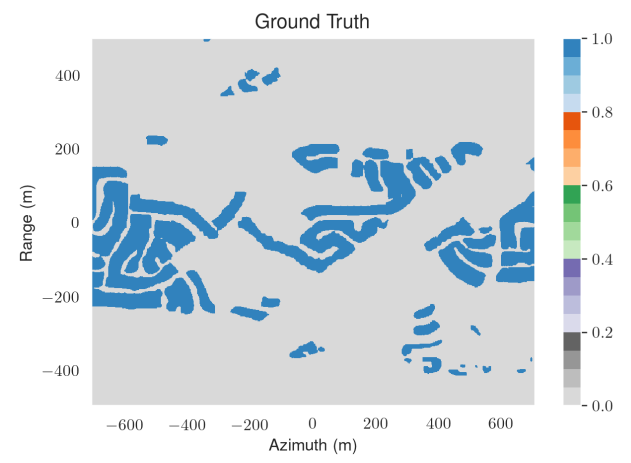

(a) Scene 1

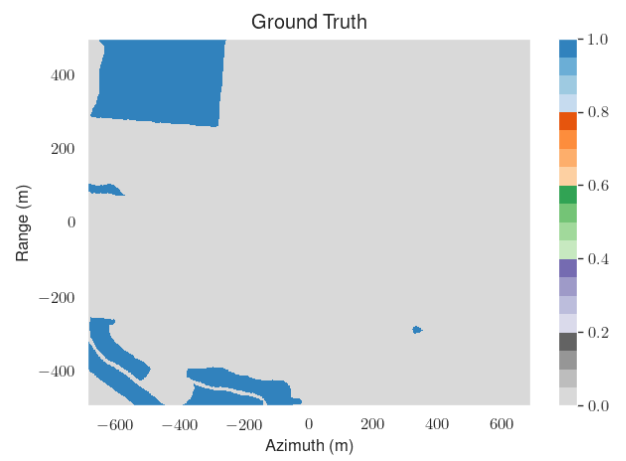

(b) Scene 2

Fig. 4: Ground truth for scenes 1 and 2

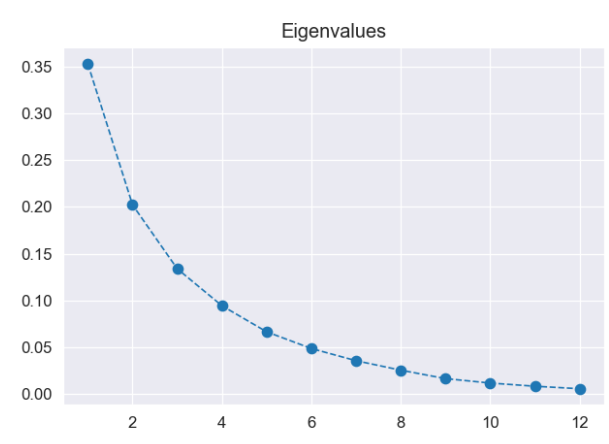

Fig. 5: Repartition of eigenvalues mean over the ITS for the scene 1 .

3) Influence of the estimation of $\sigma^{2}$ : In [1], the noise variance $\sigma^{2}$ is pre-estimated locally with the mean of the $(p-R)$ lowest eigenvalues of the sample covariance matrix of all samples in the patch. This value is then used as a known parameter in the detector (cf. (21)). Figure 13 compares this method with the fully adaptive one (proposed in this paper). It shows that the parameter $\sigma^{2}$ can be left as a degree of freedom at each $t$ without losing in terms of CD performance.

\section{CONCLUSION}

This paper derived a new GLRT based on covariance matrix equality testing. This GLRT was derived assuming that the samples follow a compound Gaussian distribution with a low-rank structured covariance matrix. Experiments on two UAVSAR scenes showed the interest of combining both the non-Gaussian approach and the structure prior information for CD in SAR-ITS. In this work, the rank of the model is assumed to be fixed and estimated at a previous stage. The robustness of the proposed detector to this rank selection was also illustrated in the experiments. A potential extension of this work would be to investigate the change of the rank within such a CD process.

\section{REFERENCES}

[1] A. Mian, A. Breloy, G. Ginolhac, and J.-P. Ovarlez, "Robust low-rank change detection for SAR image time series," in IGARSS $2019-2019$
IEEE International Geoscience and Remote Sensing Symposium, July 2019, pp. 10079-10082.

[2] M. Hussian, D. Chen, A. Cheng, H. Wei, and D. Stanley, "Change detection from remotely sensed images: From pixel-based to objectbased approaches," ISPRS Journal of photogrammetry and remote sensing, vol. 80, pp. 91-106, 2013.

[3] M. Preiss, D. A. Gray, and N. J. S. Stacy, "Detecting scene changes using synthetic aperture radar interferometry," IEEE Transactions on Geoscience and Remote Sensing, vol. 44, no. 8, pp. 2041-2054, Aug 2006.

[4] Y. Bazi, L. Bruzzone, and F. Melgani, "An unsupervised approach based on the generalized Gaussian model to automatic change detection in multitemporal SAR images," IEEE Transactions on Geoscience and Remote Sensing, vol. 43, no. 4, pp. 874-887, April 2005.

[5] A. Mian, J.-P. Ovarlez, A. M. Atto, and G. Ginolhac, "Design of new wavelet packets adapted to high-resolution SAR images with an application to target detection," IEEE Transactions on Geoscience and Remote Sensing, 2019.

[6] K. Conradsen, A. A. Nielsen, J. Schou, and H. Skriver, "A test statistic in the complex Wishart distribution and its application to change detection in polarimetric SAR data," IEEE Transactions on Geoscience and Remote Sensing, vol. 41, no. 1, pp. 4-19, Jan 2003.

[7] L. M. Novak, "Coherent change detection for multi-polarization SAR," in Conference Record of the Thirty-Ninth Asilomar Conference onSignals, Systems and Computers, 2005., Oct 2005, pp. 568-573.

[8] J. Barber, "A generalized likelihood ratio test for coherent change detection in polarimetric SAR," IEEE Geoscience and Remote Sensing Letters, vol. 12, no. 9, pp. 1873-1877, Sept 2015.

[9] V. Carotenuto, A. De Maio, C. Clemente, and J. Soraghan, "Unstructured versus structured GLRT for multipolarization SAR change detection," IEEE Geoscience and Remote Sensing Letters, vol. 12, no. 8, pp. 16651669, Aug 2015.

[10] D. Ciuonzo, V. Carotenuto, and A. De Maio, "On multiple covariance equality testing with application to SAR change detection," IEEE Transactions on Signal Processing, vol. 65, no. 19, pp. 5078-5091, Oct 2017.

[11] A. De Maio, D. Orlando, L. Pallotta, and C. Clemente, "A multifamily GLRT for oil spill detection," IEEE Transactions on Geoscience and Remote Sensing, vol. 55, no. 1, pp. 63-79, Jan 2017.

[12] D. Ratha, S. De, T. Celik, and A. Bhattacharya, "Change detection in polarimetric SAR images using a geodesic distance between scattering mechanisms," IEEE Geoscience and Remote Sensing Letters, vol. 14, no. 7, pp. 1066-1070, July 2017.

[13] A. D. C. Nascimento, A. C. Frery, and R. J. Cintra, "Detecting changes in fully polarimetric sar imagery with statistical information theory," IEEE Transactions on Geoscience and Remote Sensing, vol. 57, no. 3, pp. 1380-1392, 2019.

[14] H. Nagao, "On some test criteria for covariance matrix," Ann. Statist., vol. 1, no. 4, pp. 700-709, 071973.

[15] J. R. Schott, "Some tests for the equality of covariance matrices," Journal of Statistical Planning and Inference, vol. 94, no. 1, pp. 25 - 36, 2001.

[16] T. W. Anderson, An Introduction to Multivariate Statistical Analysis, Wiley Series in Probability and Statistics. Wiley, 2003. 


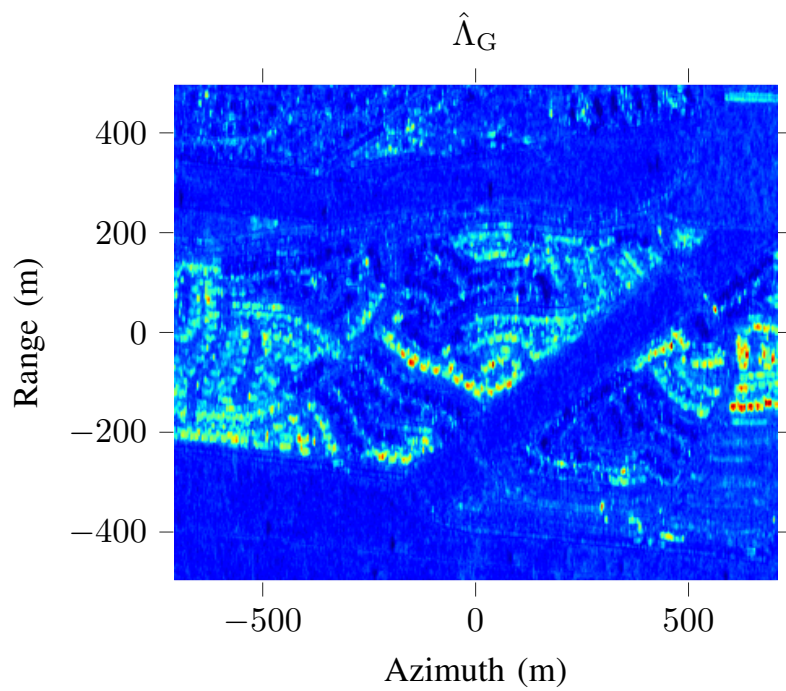

(a) Gaussian

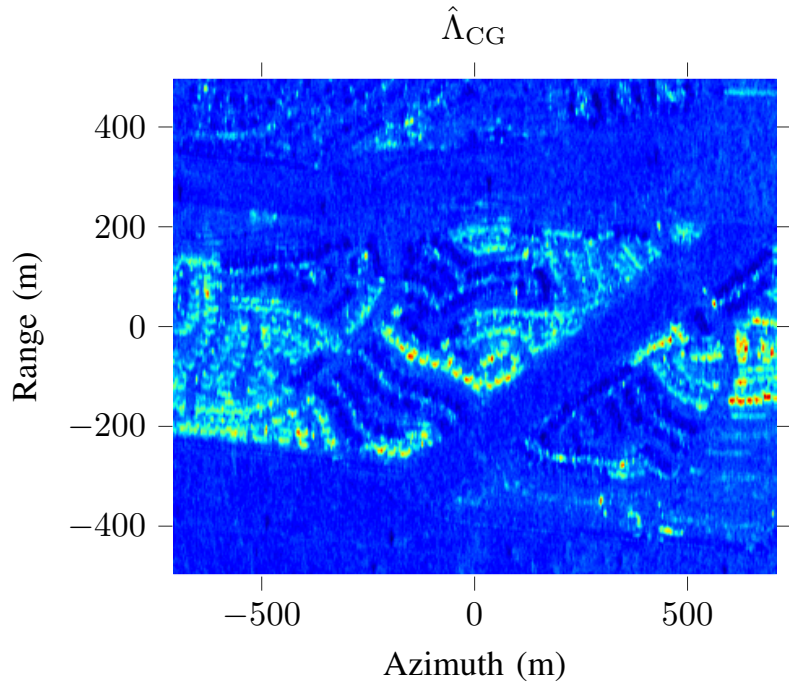

(c) Compound Gaussian

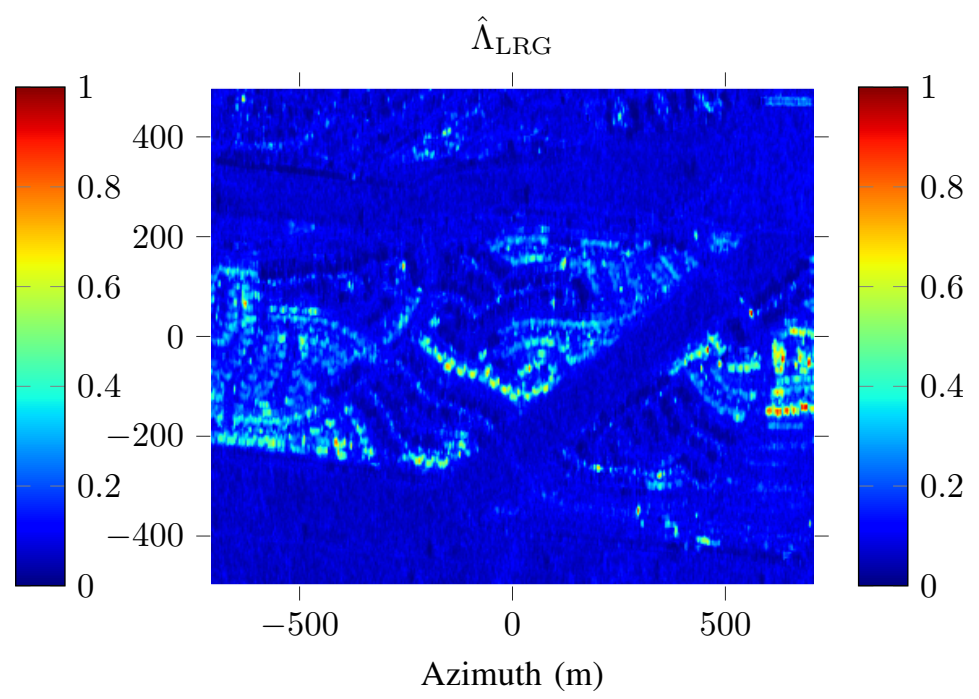

(b) Low Rank Gaussian

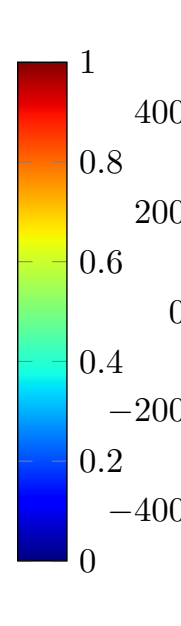

$-400$

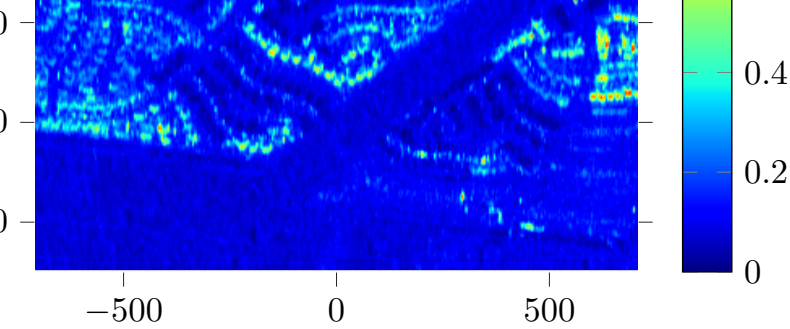

Azimuth (m)

Fig. 6: Outputs of the 4 methods for the scene 1: Gaussian, Low Rank Gaussian, Compound Gaussian (CG) and Low Rank Compound Gaussian (LRCG). Rank is fixed as 3, the window size is $7 \times 7$ and $\sigma^{2}$ is assumed unknown for low rank models.

[17] M. Hallin and D. Paindaveine, "Optimal tests for homogeneity of covariance, scale, and shape," Journal of Multivariate Analysis, vol. 100, no. 3, pp. $422-444,2009$.

[18] P. Galeano and D. Peña, "Covariance changes detection in multivariate time series," Journal of Statistical Planning and Inference, vol. 137, no. 1, pp. $194-211,2007$.

[19] A. Aue, S. Hörmann, L. Horváth, and M. Reimherr, "Break detection in the covariance structure of multivariate time series models," Ann. Statist., vol. 37, no. 6B, pp. 4046-4087, 122009.

[20] M. Greco and F. Gini, "Statistical analysis of high-resolution SAR ground clutter data," IEEE Transactions on Geoscience and Remote Sensing, vol. 45, no. 3, pp. 566-575, 2007.

[21] E. Ollila, D. E. Tyler, V. Koivunen, and H. V. Poor, "Complex elliptically symmetric distributions: Survey, new results and applications," IEEE Transactions on Signal Processing, vol. 60, no. 11, pp. 5597-5625, Nov 2012.

[22] P. Formont, F. Pascal, G. Vasile, J.-P. Ovarlez, and L. Ferro-Famil, "Statistical classification for heterogeneous polarimetric SAR images," IEEE Journal of Selected Topics in Signal Processing, vol. 5, no. 3, pp. 567-576, June 2011.
[23] M. Liu, H. Zhang, and C. Wang, "Change detection in urban areas of high-resolution polarization SAR images using heterogeneous clutter models," in 2011 3rd International Asia-Pacific Conference on Synthetic Aperture Radar (APSAR), Sept 2011, pp. 1-4.

[24] A. Mian, G. Ginolhac, and J.-P. Ovarlez, "Robust low-rank change detection for SAR image time series," in 2019 IEEE International Geoscience and Remote Sensing Symposium (IGARSS), 2019.

[25] A. Mian, G. Ginolhac, J.-P. Ovarlez, and A. M. Atto, "New robust statistics for change detection in time series of multivariate SAR images," IEEE Transactions on Signal Processing, vol. 67, no. 2, pp. 520-534, Jan 2019.

[26] M. E Tipping and C. M. Bishop, "Probabilistic principal component analysis," Journal of the Royal Statistical Society: Series B (Statistical Methodology), vol. 61, no. 3, pp. 611-622, 1999.

[27] B. Kang, V. Monga, and M. Rangaswamy, "Rank-constrained maximum likelihood estimation of structured covariance matrices," IEEE Transactions on Aerospace and Electronic Systems, vol. 50, no. 1, pp. 501-515, January 2014.

[28] R. Ben Abdallah, A. Mian, A. Breloy, M. N. El Korso, and D. Lautru, "Detection methods based on structured covariance matrices for multi- 


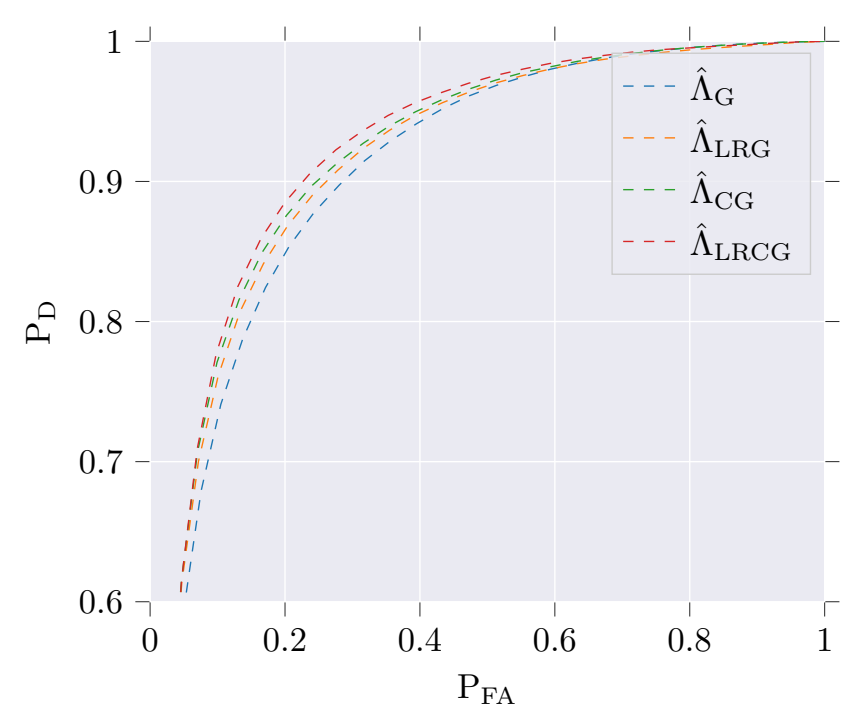

Fig. 7: Comparison between 4 methods for the scene 1: Gaussian, Low Rank Gaussian, Compound Gaussian (CG) and Low Rank Compound Gaussian (LRCG). Rank is fixed as 3, the window size is $7 \times 7$ and $\sigma^{2}$ is assumed unknown for both low rank models.

variate SAR images processing," IEEE Geoscience and Remote Sensing Letters, 2019.

[29] I. S. Reed, J. D. Mallett, and L. E. Brennan, "Rapid convergence rate in adaptive arrays," IEEE Transactions on Aerospace and Electronic Systems, vol. AES-10, no. 6, pp. 853 - 863, November 1974.

[30] A. Breloy, G. Ginolhac, F. Pascal, and P. Forster, "Clutter subspace estimation in low rank heterogeneous noise context," IEEE Transactions on Signal Processing, vol. 63, no. 9, pp. 2173-2182, May 2015.

[31] A. Breloy, G. Ginolhac, F. Pascal, and P. Forster, "Robust covariance matrix estimation in heterogeneous low-rank context," IEEE Transactions on Signal Processing, vol. 64, no. 22, pp. 5794 - 5806, November 2016.

[32] Y. Sun, P. Babu, and D. P. Palomar, "Robust estimation of structured covariance matrix for heavy-tailed elliptical distributions," IEEE Transactions on Signal Processing, vol. 64, no. 14, pp. 3576 - 3590, July 2016.

[33] Y. Sun, A. Breloy, P. Babu, D. P. Palomar, F. Pascal, and G. Ginolhac, "Low-complexity algorithms for low rank clutter parameters estimation in radar systems," Signal Processing, IEEE Transactions on, vol. 64, no. 8, pp. 1986 - 1998, April 2016.

[34] R. B. Abdallah, A. Breloy, A. Taylor, M. N. El Korso, and D. Lautru, "Signal subspace change detection in structured covariance matrices," in 2019 27th European Signal Processing Conference (EUSIPCO), Sep. 2019, pp. 1-5.

[35] F. Pascal, Y. Chitour, J.-P. Ovarlez, P. Forster, and P. Larzabal, "Covariance structure maximum-likelihood estimates in compound Gaussian noise: Existence and algorithm analysis," IEEE Transactions on Signal Processing, vol. 56, no. 1, pp. 34-48, Jan 2008.

[36] P. Stoica and Y. Selen, "Model-order selection: a review of information criterion rules," IEEE Signal Processing Magazine, vol. 21, no. 4, pp. 36-47, July 2004.

[37] A. D. C. Nascimento, A. C. Frery, and R. J. Cintra, "Detecting changes in fully polarimetric sar imagery with statistical information theory," arXiv:1801.08901, 2018.

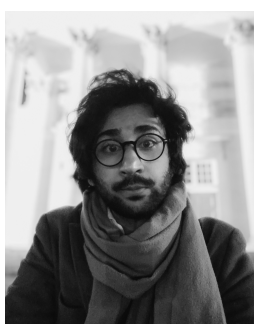

Ammar Mian (S'18) received the master's degree in signal processing from Grenoble INP, Grenoble, France, in 2016 and the Ph.D. degree in signal processing from CentraleSupélec, Gif-sur-Yvette, France in 2019 . He is currently a post-doctoral researcher at Aalto University, Espoo, Finland. His research interests include statistical signal processing, Riemannian geometry, time-frequency analysis, and machine learning.

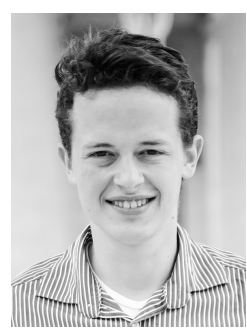

Antoine Collas received a master's degree in Computer Sciences and Engineering with a specialty "Decision Data Mining" from UTC, Compiègne, France in 2019. He is currently a Ph.D student in signal and image processing at CentraleSupélec, Gifsur-Yvette, France. His research interests include statistical signal processing, Riemannian geometry and machine learning.

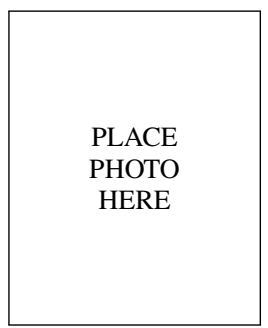

Arnaud Breloy (S'14-M'16) received the graduate degree from Ecole Centrale Marseille, Marseille, France, the master's degree in signal and image processing from the University of Aix-Marseille, Provence, France, in 2012-2013 and the Ph.D degree in signal processing from the University of Paris-Saclay, Saint-Aubin, France, in 2015. The thesis was done in collaboration between SATIE (ENS Cachan) and SONDRA (CentraleSupélec) laboratories. Since 2016, he has been an Associate Professor with the LEME laboratory, University Paris Nanterre, Nanterre, France. His research interests include statistical signal processing, array and radar processing, robust estimation methods, and subspace-based methods.

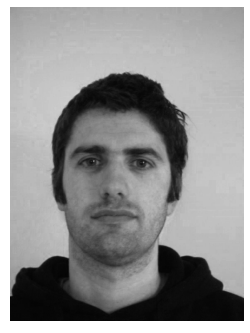

Guillame Ginolhac (S'98,M'01,SM'12) received the Master's degree of Electrical Engineer, in 1997 and the Ph.D. degree of Signal Processing, in 2001 both from the Grenoble-INP university (France). From 2002 to 2012, he was an associate professor in SATIE Lab and University Paris Nanterre. In 2011, he obtained a Research Directorship Habilitation (HDR) thesis in Signal Processing from the ENS Cachan institute. From 2012, Guillaume Ginolhac is a full professor in LISTIC Lab at University Savoie Mont-Blanc. From July 2018, he is the head of the "Learning, Fusion and Remote Sensing" group of LISTIC. His research interests include estimation and detection theory for statistical signal processing and applications to array processing and radar remote sensing. 


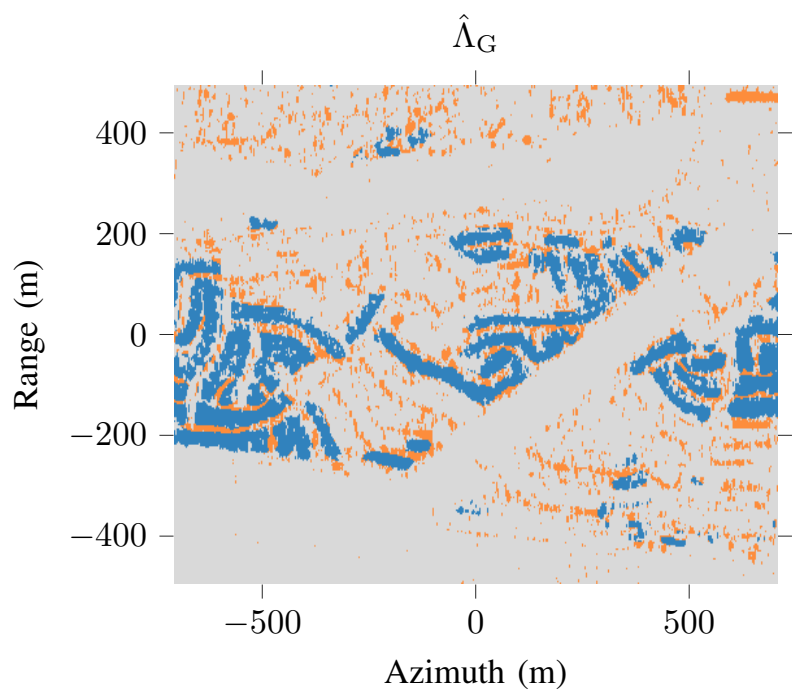

(a) Gaussian

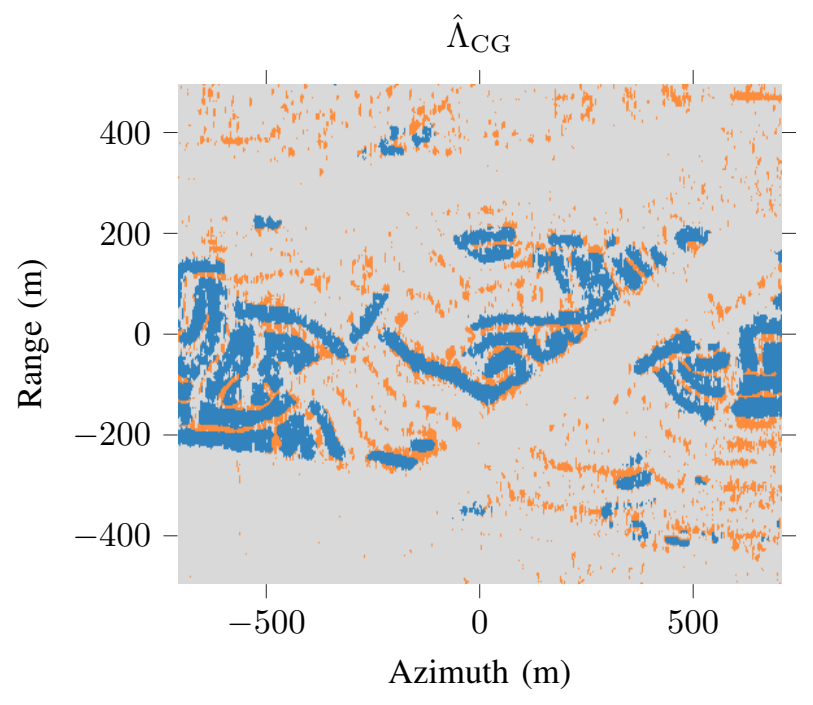

(c) Compound Gaussian

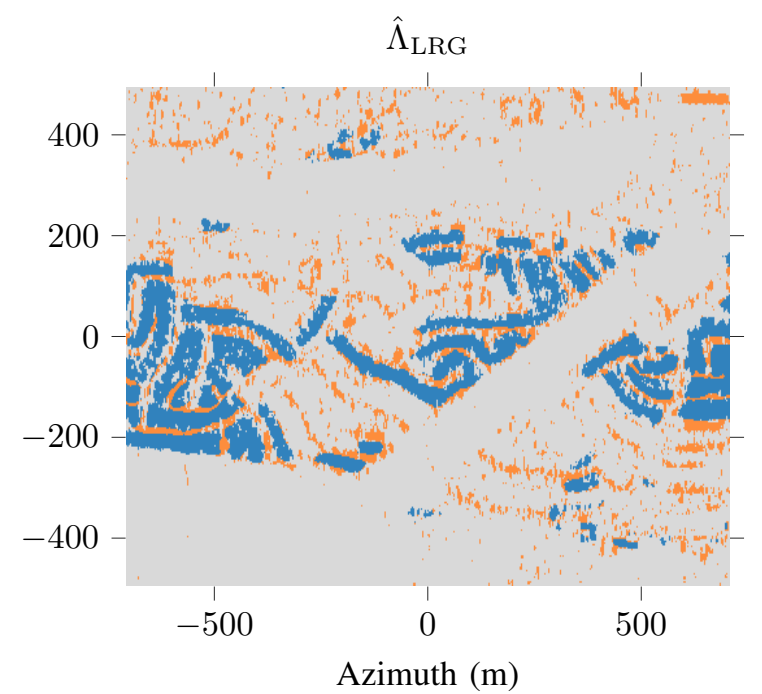

(b) Low Rank Gaussian

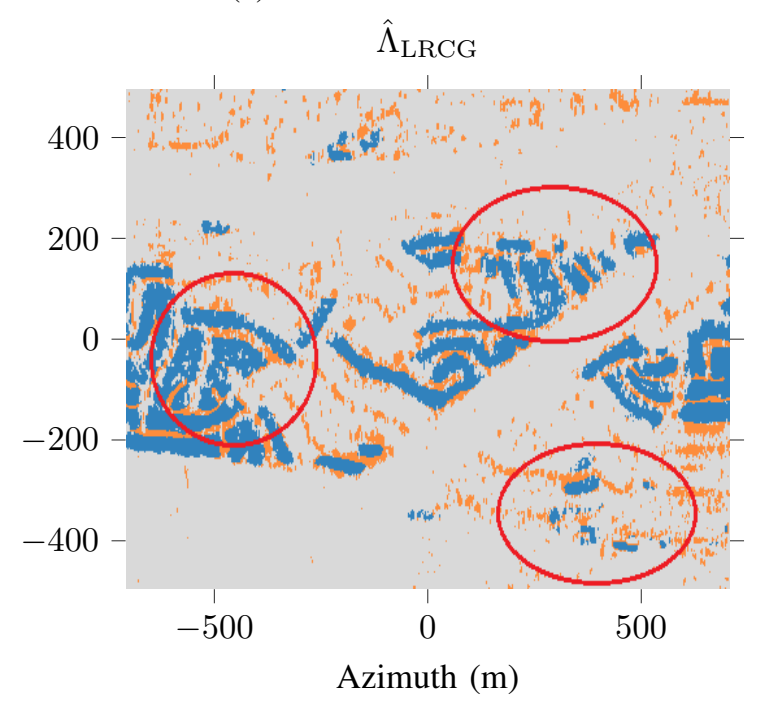

(d) Low Rank Compound Gaussian

Fig. 8: Thresholded outputs of the 4 methods for the scene 1 for a fixed probability of false alarm (10\%): true detection in blue and false alarms in orange. Rank is fixed as 3, the window size is $7 \times 7$ and $\sigma^{2}$ is assumed unknown for both low rank models.

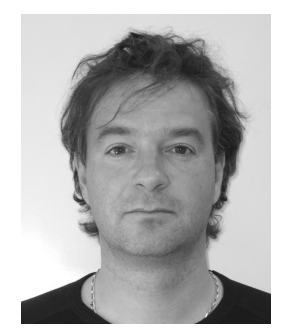

Jean-Philippe Ovarlez (M'06) was born in Denain, France in 1963. He received jointly the engineering degree from Ecole Supérieure d'Electronique Automatique et Informatique (ESIEA), Paris, France and the Diplôme d'Etudes Approfondies degree in Signal Processing from University of Paris XI, Orsay, France and the Ph.D. degree in Physics from the University of Paris VI, Paris, France, in 1987 and 1992, respectively. In 2011, he obtained a Research Directorship Habilitation (HDR) thesis in Signal Processing from the University of Paris-Sud and his qualification to the University Professor position. In 1992, he joined the Electromagnetic and Radar Division of the French Aerospace Lab (ONERA), Palaiseau, France, where he is currently Director of Research and member of the Scientific Committee of the ONERA Physics domain. Since 2008, he is attached at a part time to CentraleSupélec SONDRA Lab, in charge of Signal Processing activities supervision. In 2015, he becomes member of Special Area Team (SAT) in Theoretical and Methodological Trends in Signal Processing (TMTSP), EURASIP and treasurer of the IEEE GRSS French Chapter in 2016. His research interests are centered in the topic of Statistical Signal Processing for radar and SAR applications such as Time-Frequency, imaging, detection and parameters estimation. 


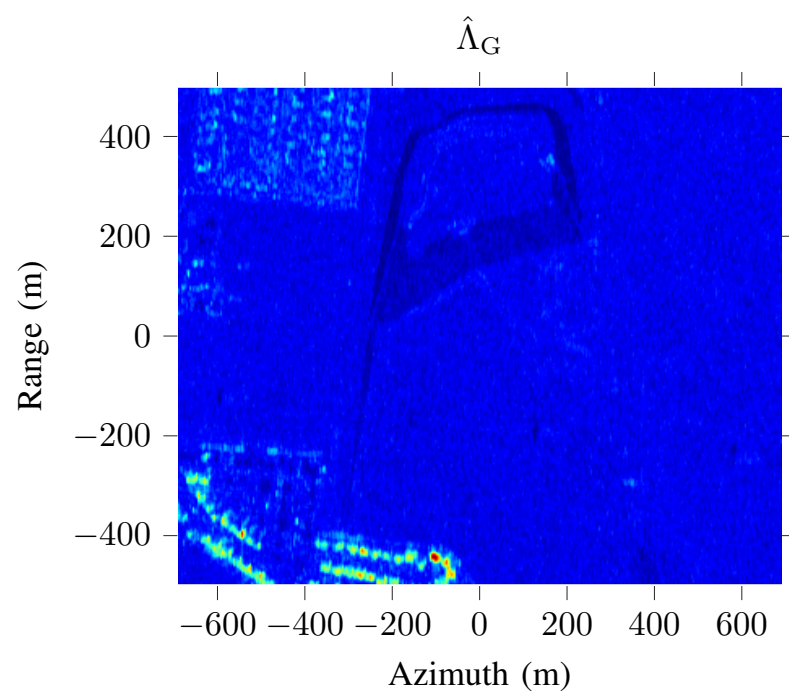

(a) Gaussian

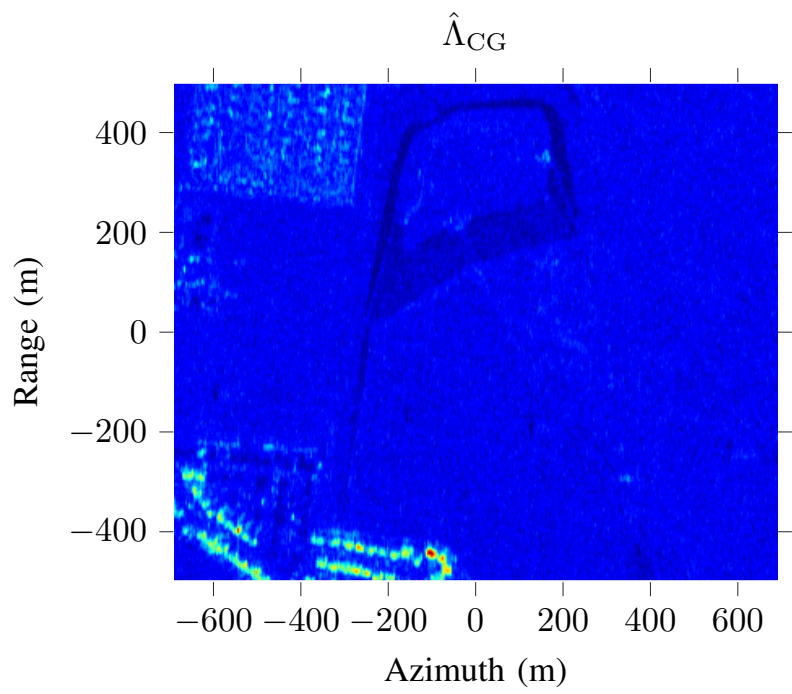

(c) Compound Gaussian

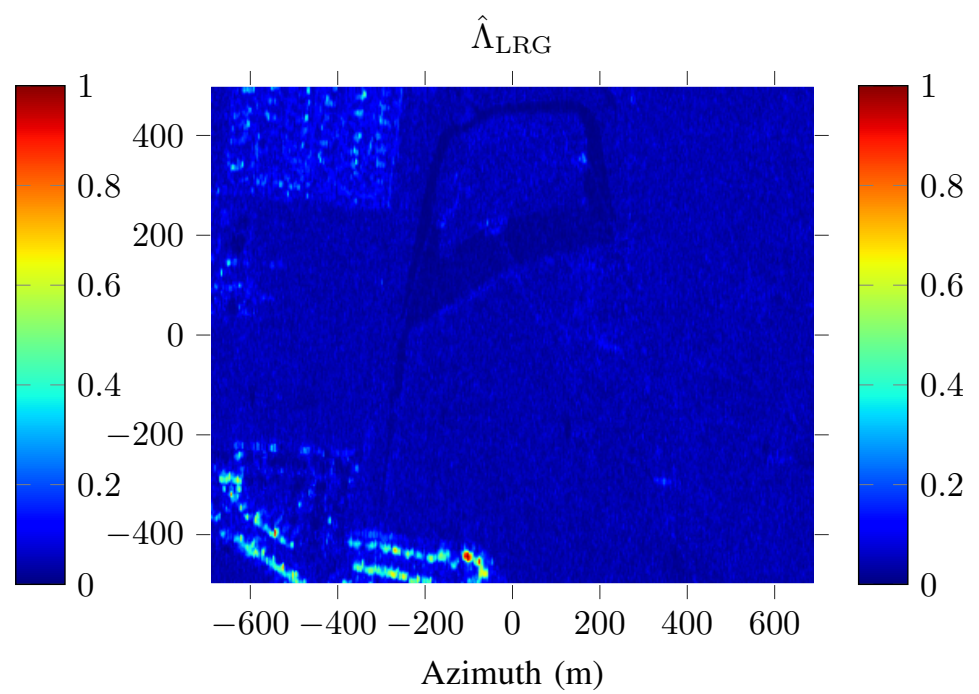

(b) Low Rank Gaussian

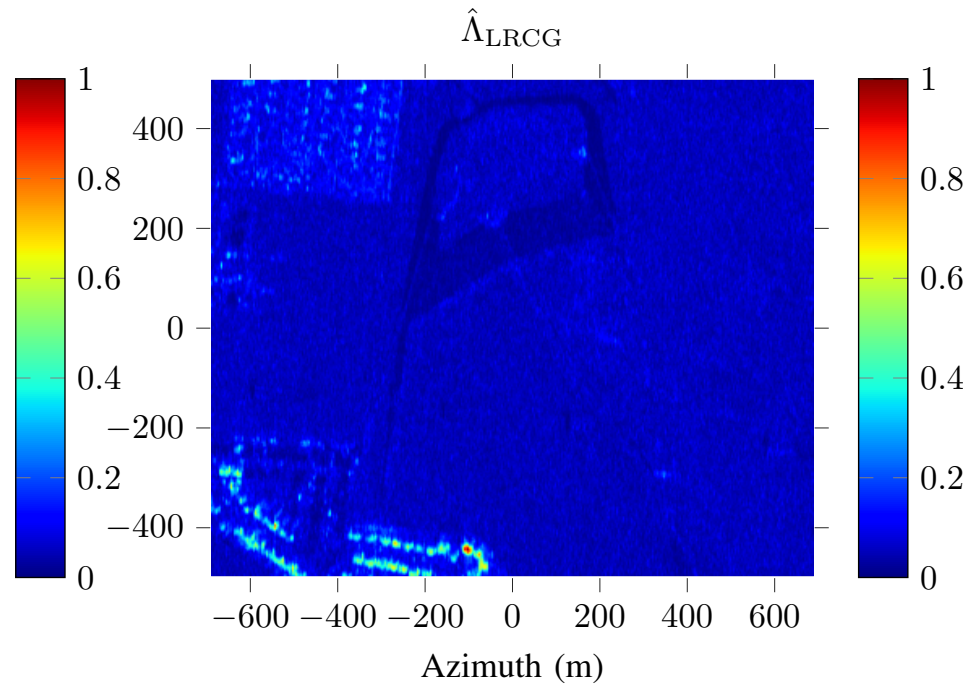

(d) Low Rank Compound Gaussian

Fig. 9: Outputs of the 4 methods for the scene 2: Gaussian, Low Rank Gaussian, Compound Gaussian (CG) and Low Rank Compound Gaussian (LRCG). Rank is fixed as 3, the window size is $7 \times 7$ and $\sigma^{2}$ is assumed unknown for low rank models. 


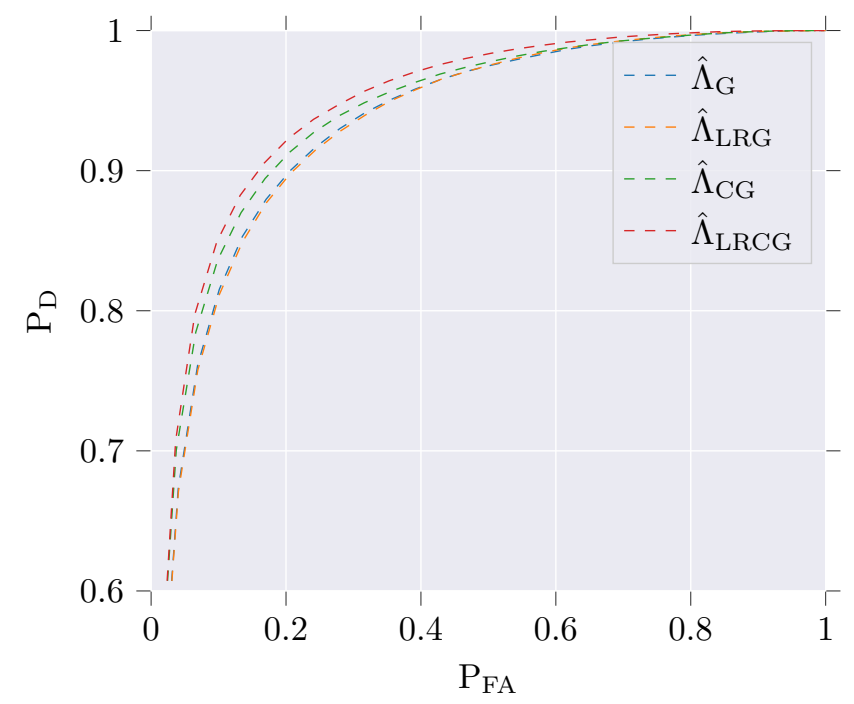

Fig. 10: Comparison between 4 methods for the scene 2: Gaussian, Low Rank Gaussian, Compound Gaussian (CG) and Low Rank Compound Gaussian (LRCG). Rank is fixed as 3, the window size is $7 \times 7$ and $\sigma^{2}$ is assumed unknown for low rank models. 


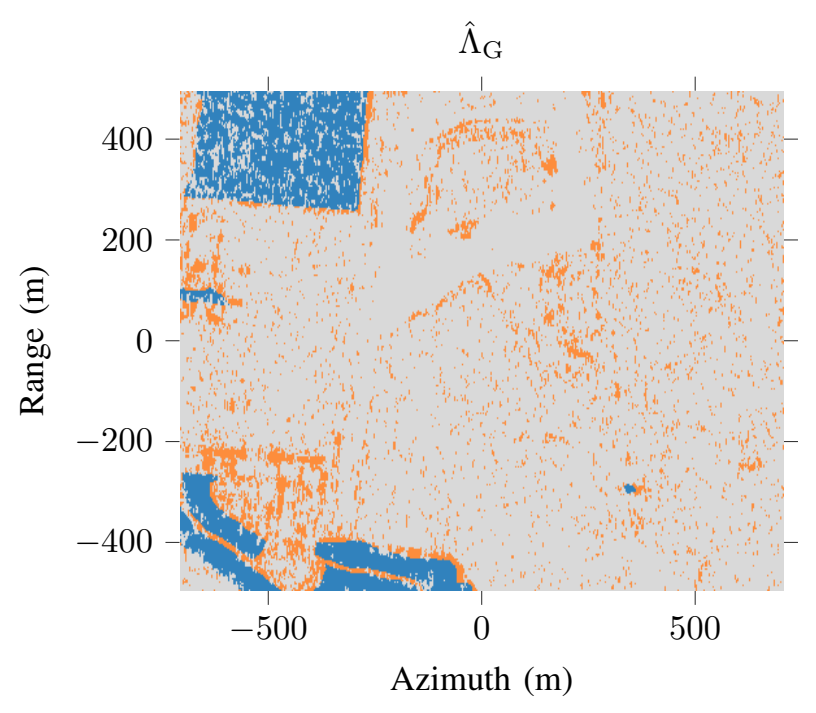

(a) Gaussian

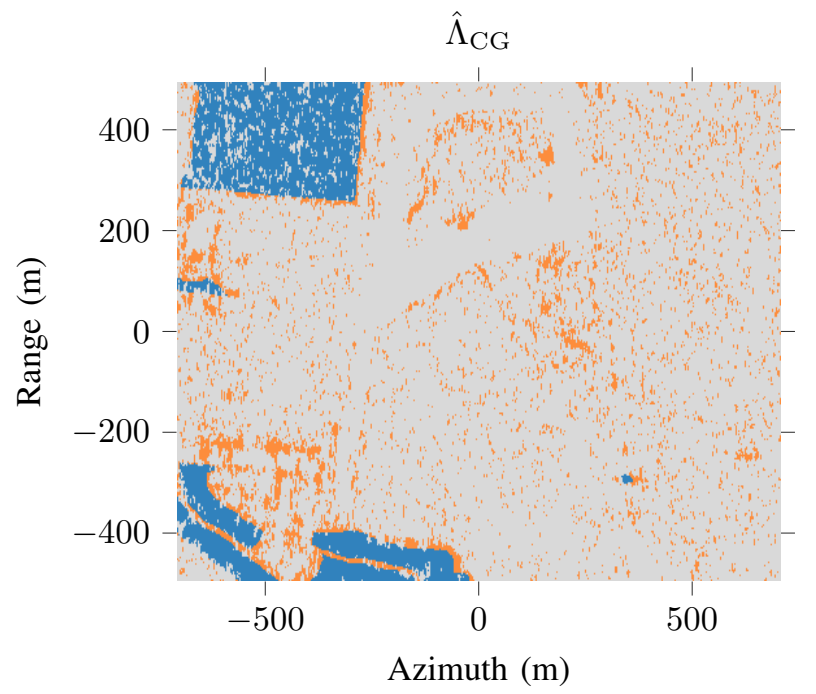

(c) Compound Gaussian

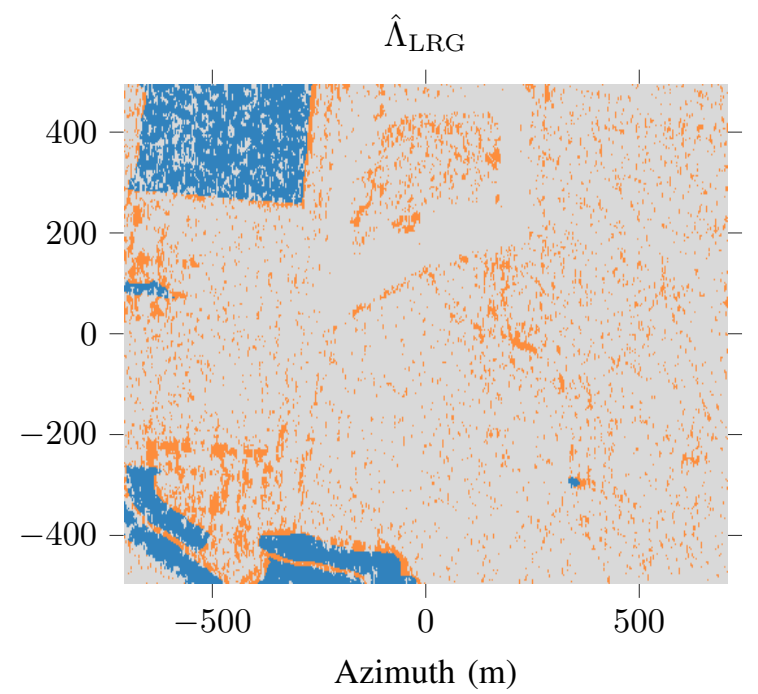

(b) Low Rank Gaussian

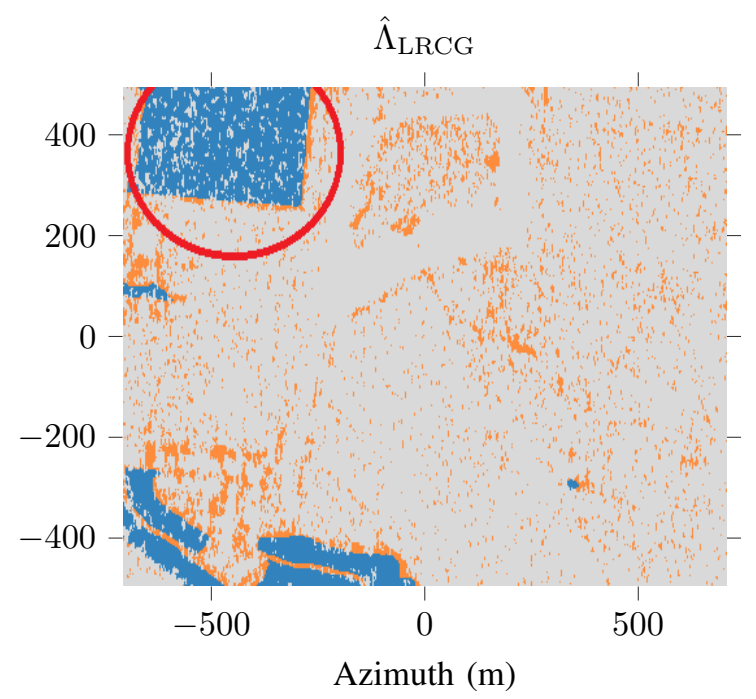

(d) Low Rank Compound Gaussian

Fig. 11: Thresholded outputs of the 4 methods for the scene 1 for a fixed probability of false alarm (10\%): true detection in blue and false alarms in orange. Rank is fixed as 3, the window size is $7 \times 7$ and $\sigma^{2}$ is assumed unknown for both low rank models. 


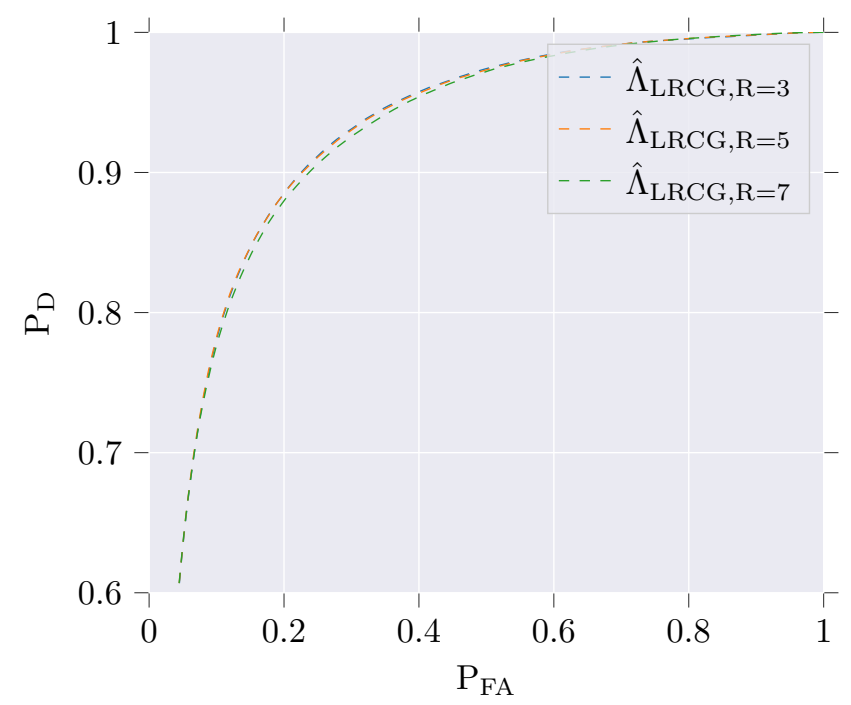

Fig. 12: Comparison for the scene 1 of the Low Rank Compound Gaussian (LRCG) for different rank values. The window size is fixed at $7 \times 7$ and $\sigma^{2}$ is assumed unknown.

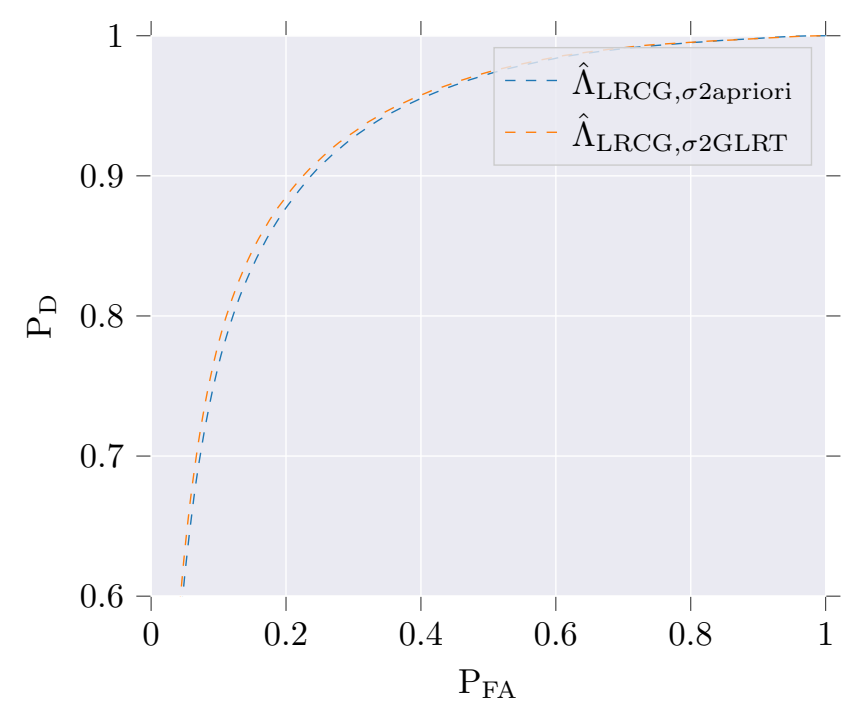

Fig. 13: LRCG: comparison of ROC curves between assumed known $\sigma^{2}$ and assumed unknown $\sigma^{2}$ (integrated in GLRT). $R=3$, Window size is equal to $7 \times 7$. 